\title{
Decomposition of time-covariant operations on quantum systems with continuous and/or discrete energy spectrum
}

\author{
Dominik Janzing* \\ IAKS Prof. Beth, Arbeitsgruppe Quantum Computing, \\ Universität Karlsruhe, \\ Am Fasanengarten 5, D-76 131 Karlsruhe, Germany
}

Sept 30, 2004

\begin{abstract}
Every completely positive map $G$ that commutes which the Hamiltonian time evolution is an integral or sum over (densely defined) CPmaps $G_{\sigma}$ where $\sigma$ is the energy that is transferred to or taken from the environment. If the spectrum is non-degenerated each $G_{\sigma}$ is a dephasing channel followed by an energy shift. The dephasing is given by the Hadamard product of the density operator with a (formally defined) positive operator. The Kraus operator of the energy shift is a partial isometry which defines a translation on $\mathbb{R}$ with respect to a non-translation-invariant measure.

As an example, I calculate this decomposition explicitly for the rotation invariant gaussian channel on a single mode.

I address the question under what conditions a covariant channel destroys superpositions between mutually orthogonal states on the same orbit. For channels which allow mutually orthogonal output states on the same orbit, a lower bound on the quantum capacity is derived using the Fourier transform of the CP-map-valued measure $\left(G_{\sigma}\right)$.
\end{abstract}

*e-mail: janzing@ira.uka.de 


\section{Introduction}

It was an important insight in the early days of quantum computing and quantum cryptography research that quantum theory should not be considered as a purely physical theory, but it rather defines a new kind of information, called quantum information. The decisive feature is its fragility since quantum information is destroyed whenever one tries to copy it [1].

The question which physical processes and information channels preserve quantum information plays therefore a crucial role in the theory of quantum communication. A central concept for addressing this issue is the notion of a channel. Here we consider a channel or ("operation") to be a completely positive trace preserving map $\rho \mapsto G(\rho)$ where $\rho$ and $G(\rho)$ are positive operators of trace one acting on Hilbert spaces $\mathcal{H}_{\text {in }}$ and $\mathcal{H}_{\text {out }}$, respectively [2]. Analyzing quantum or classical channel capacities is in general a difficult task. Here we consider a certain kind of channels or operations, namely those which are time-covariant. For simplicity, we will assume that the input and the output state is a state of the same physical system. Then we have $\mathcal{H}_{\text {in }}=\mathcal{H}_{\text {out }}=: \mathcal{H}$. Furthermore we have a Hamiltonian time evolution on $\mathcal{H}$ generated by a densely defined self-adjoint operator $H$ which reads

$$
\alpha_{t}(\rho):=e^{-i H t} \rho e^{i H t}
$$

where $\rho$ is a positive operator with trace one. We call a channel $G$ timecovariant $^{1}$ if it satisfies the equation

$$
\alpha_{t} \circ G=G \circ \alpha_{t}
$$

It is a special case of the requirement

$$
G\left(U_{g} \rho U_{g}^{\dagger}\right)=U_{g} G(\rho) U_{g}^{\dagger}
$$

where $g \mapsto U_{g}$ is an arbitrary unitary group representation.

For compact groups, each covariant CP-map $G$ has a representation (see [3] which refers partly to [4])

$$
G(\rho)=\sum_{j} L_{j} \rho L_{j}^{\dagger}
$$

\footnotetext{
${ }^{1}$ Note that in the context of classical linear systems the corresponding property is usually referred to time- "invariance".
} 
where the Kraus operators $L_{j}$ satisfy

$$
U_{g} L_{j} U_{g}^{\dagger}=\sum_{k} d_{j k}(g) L_{k}
$$

and $g \mapsto\left[d_{j k}(g)\right]_{j, k \leq d}$ is a $d$-dimensional unitary representation with arbitrary $d$ (in [3] it is mainly focussed on the case that $g \mapsto U_{g}$ is an irreducible representation). Note that the time evolution can only be described by a compact group if it is periodic which cannot be guaranteed even in the finite dimensional case. In [5] the case is considered that $G$ is replaced by a whole dynamical semi-group $\left(G_{t}\right)_{t \in \mathbb{R}^{+}}$which is covariant with respect to a group representation.

Condition (2) appears naturally, for instance, in the following situations:

1. Theory of timing information: Assume that the density matrix $\rho$ is subjected to an arbitrary quantum operation $G$ at a completely unknown time instant. Then the statistical description of this operation leads to a map $G^{\prime}$ which satisfies necessarily (2). This is the key idea in [6] where condition (2) defines a "quasi-order of clocks" which classifies systems with respect to their timing information. The time-covariant maps are exactly those which can be implemented without using external clocks. Given a non-stationary state $\rho$, the set of states which can be obtained from $\rho$ using time-covariant operations are those which have at most as much timing information as $\rho$.

2. Decoherence: Dephasing of systems is described by a decay of the offdiagonal entries with respect to the energy eigenbasis. This channels satisfy clearly condition (2).

3. Scattering processes / Quantum generalization of transfer functions: Time-covariant operations appear naturally in the description of scattering processes. Then $G$ generalizes the scattering operator by including classical or quantum stochastic fluctuations. Here scattering is understood in a rather general sense. Apart from the situation that a particle is scattered by the potential of another particle, one may, for instance, also think of a light beam that passes a filter. One may consider time-covariant maps as quantum generalization of classical time-invariant linear devices which are described by their transfer functions. 
The paper is organized as follows. In Section 2 we recall briefly how to describe classical time-invariant linear systems in signal processing by a transfer function [7]. To figure out to what extent this concept can be generalized to the quantum stochastic setting is the key motivation of this paper. In Section 3 we recall abstract scattering theory in Hilbert spaces and explain in Section 4 why time-covariant channels are also considered as a natural generalization of scattering theory. In Section 5 we consider CP-maps which are given by the Hadamard product of the density matrix with a positive matrix. These maps will turn out as a building block of our decomposition. In Section 6 we derive the general form of a time-covariant CP-map. The main result is that every time-covariant CP-map acting on a system with non-degenerate Hamiltonian has a (densely defined) decomposition as an integral or sum over (densely defined) CP-maps $G_{\sigma}$ is a dephasing followed by an energy shift. In Section 7 we address the question whether and to what extent covariant channels can destroy superpositions between states on the same orbit (with respect to the time-evolution). We use the general decomposition to derive a lower bound on the quantum capacity of a specific type of time-covariant channels, namely those which have as output mutually orthogonal density

operators on the same orbit. In Section 8 we apply the decomposition to a single mode gaussian channel.

\section{Classical time-invariant linear systems}

To show the differences and the analogies to the theory of classical linear time-invariant channels we briefly rephrase the concept of transfer functions which is among the most important tools in classical signal processing.

Consider a classical channel with an incoming and an outgoing signal. These may, for instance, be light pulses or electrical pulses, acoustical or other signals. Let $t \mapsto Y(t)$ be the incoming signal where $Y(t)$ is the value of an arbitrary physical quantity at the time instant $t$. Let $Y^{\prime}(t)$ denote the outgoing signal. In order to avoid problems with undefined Fourier transforms we assume that $Y$ and $Y^{\prime}$ are vectors in $L^{2}(\mathbb{R})$, the Hilbert space of square-integrable functions on $\mathbb{R}$. We assume that the system is described by a linear bounded operator $A$ on $L^{2}(\mathbb{R})$ with

$$
Y^{\prime}=A Y
$$


Assuming time-invariance of the channel we have $Y^{\prime}(t+s)=(A Y)(t+s)$. Defining the group of shifts $\left(S_{t}\right)_{t \in \mathbb{R}}$ by

$$
\left(S_{t} Y\right)(s)=Y(t-s)
$$

we have

$$
S_{t} A=A S_{t} \forall t \in \mathbb{R}
$$

The implications of this condition can easily be derived by applying Fourier transformation to both sides of the equation: The shift operators $S_{t}$ act then as the multiplication operators $M_{t}$

$$
\hat{Y} \mapsto M_{t} \hat{Y}
$$

with

$$
\left(M_{t} \hat{Y}\right)(\omega)=e^{i \omega t} \hat{Y}(\omega) .
$$

A linear operator commuting with all operators $M_{t}$ is necessarily a multiplication operator as well (see [8], 12.1.5). Therefore $A$ can be characterized by a so-called transfer function $a$ with

$$
\hat{Y}^{\prime}(\omega)=a(\omega) \hat{Y}(\omega) .
$$

Note that it is essential that the quantity $Y(t)$ is a scalar. If $Y(t)$ is a vector of dimension $d$ greater than 1 the spectrum of the time-evolution group $S_{t}$ is degenerated and $\hat{Y}(\omega)$ is an element of a vector space $\mathbb{C}^{d}$ or $\mathbb{R}^{d}$ and the transfer function $a$ had to be replaced by a $d \times d$-matrix. It is straightforward to ask whether time-covariant quantum operations allow a natural generalization of the transfer function. However, one should recall that the situation with non-degenerated spectrum is possible on the Hilbert space level (this is well-known in abstract scattering theory, as will be rephrased in the next section) but not on the level of density matrices, where the dynamics is generated by the super-operator $-i[H,$.$] . The latter has always degenerated$ spectrum.

\section{Abstract scattering theory}

A similar approach as above applies to a scattering process of a quantum mechanical particle: A particle which comes from the infinity and passes a 
scattering potential. After it has left the potential it disappears to infinity. Here we only recall some standard results of scattering theory $[9,8]$ which are essential for this paper. Let $H=H_{i}+H_{0}$ be the total Hamiltonian of the system which consists of the free Hamiltonian $H_{0}$ and the interactions term $H_{i}$. One assumes that the particle moves approximatively according to its free Hamiltonian $H_{0}$ for $t \rightarrow \pm \infty$. For potentials which decay sufficiently with the distance between particle and the scattering center, the limits

$$
\left|\psi^{ \pm}\right\rangle:=\lim _{t \rightarrow \pm \infty} \exp (i H t) \exp \left(-i H_{0} t\right)|\psi\rangle
$$

exist [9] on an appropriate subspace of wave functions $|\psi\rangle$. There is a rich literature addressing the question under which circumstances and on which subspaces one can define a unitary scattering operator ${ }^{2}$

$$
\left|\psi^{-}\right\rangle \mapsto\left|\psi^{+}\right\rangle=: S\left|\psi_{-}\right\rangle
$$

However, if it exists it commutes with the free Hamiltonian evolution $\exp \left(-i H_{0} t\right)$. Therefore the free time evolution and the scattering operator $S$ can simultaneously be described by multiplication operators. The analogy to Section 2 can even be closer in a scattering process with so-called Lax-Phillips evolution [8]. Assume that there exist subspaces $\mathcal{H}_{-} \leq \mathcal{H}$ and $\mathcal{H}_{+} \leq \mathcal{H}$ ("incoming and outgoing subspaces", respectively) such that

$$
e^{-i H t} \mathcal{H}_{-} \subset \mathcal{H}_{-}
$$

for all negative $t$ (the particle comes from the infinity) and

$$
e^{-i H t} \mathcal{H}_{+} \subset \mathcal{H}_{+}
$$

for all positive $t$ (the particle disappears to the infinity). Assume furthermore that the intersection

$$
\cap_{t \in \mathbb{R}} \exp (-i H t) \mathcal{H}_{ \pm}
$$

vanishes and that the span of all spaces $\exp (-i H t) \mathcal{H}_{+}$as well as the span of all $\exp (-i H t) \mathcal{H}_{-}$is dense in $\mathcal{H}$. Then one can assume without loss of generality that $\mathcal{H}$ is the set of square integrable functions on $\mathbb{R}$ with respect to the Lebesgue measure, and $H$ is the multiplication operator $(H \psi)(\omega)=$ $\omega \psi(\omega)$. The scattering operator is then (like the transfer function in Section 2 ) given by $(S \psi)(\omega)=s(\omega) \psi(\omega)$ with an appropriate function $s$.

\footnotetext{
${ }^{2}$ It is usual to denote the scattering operator with $S$. On the other hand, it is usual to denote shifts by $S_{t}$ with some index $t$. He hope that this will not lead to confusions.
} 


\section{Quantum channels from scattering processes}

The unitary scattering operator $S$ in Section 3 defines a channel $G(\rho)=S \rho S^{\dagger}$ which satisfies obviously the time-covariance condition (2) with respect to the free evolution $\alpha_{t}(\rho)=\exp \left(-i H_{0} t\right) \rho \exp \left(i H_{0} t\right)$ due to $S H_{0}=H_{0} S$. It is clear that scattering with an unknown potential could lead to an operation which is a statistical mixture of maps $S \rho S^{\dagger}$. But not only classical fluctuations of the scattering potential lead to a mixture of output wave functions; due to quantum fluctuations of the potential one may also have a CP-map which is not a mixture of unitary scattering processes. To see this, consider a bipartite quantum system with Hilbert space $\mathcal{H}_{A} \otimes \mathcal{H}_{B}$ that evolves according to a joint Hamiltonian $H_{A B}$. Assume that the joint evolution coincides asymptotically with the separate evolution generated by $H_{A}+H_{B}$ and that the limits

$$
\left|\psi^{ \pm}\right\rangle:=\lim _{t \rightarrow \infty} \exp \left(i t H_{A B}\right) \exp \left(-i H_{A} t\right) \exp \left(-i H_{B} t\right)|\psi\rangle
$$

exist in an appropriate sense. Then we have formally the same situation as in Section 3 with the substitution

$$
H_{0} \mapsto H_{A}+H_{B}, \quad H \mapsto H_{A B} .
$$

If a scattering operator $U$ on $\mathcal{H}_{A} \otimes \mathcal{H}_{B}$ exists we have

$$
\left[U, H_{A}+H_{B}\right]=0
$$

Let $\rho_{A}$ be an arbitrary initial density matrix of system $A$. Then the scattering process defines a completely positive map on the system $B$ by

$$
G\left(\rho_{B}\right):=\operatorname{tr}_{A}\left(U\left(\rho_{A} \otimes \rho_{B}\right) U^{\dagger}\right) .
$$

Due to (4) it is easy to verify that $G$ is time-covariant if the initial state $\rho_{A}$ is stationary with respect to the time evolution $\exp \left(i H_{A} t\right)$. Here we do not address the difficult question in which situation the limits above exist. The problem of the existence of scattering operators in the quantum stochastic setting is addressed in [10]. This will not be our subject. The remarks above were only to show that time-covariant channels appear naturally also in the description of (possibly inelastic) scattering processes. Another situation where time-covariance appears is when the energy spectrum is discrete and a weak interaction with the environment is switched on. Then the interaction implements clearly a unitary $U$ on system + environment such that $U$ satisfies (4). 


\section{Quantum channels from Hadamard-products}

Here we consider a simple type of time-covariant CP-maps which will play a crucial role in the description of general time-covariant CP-maps.

The Hadamard product $A * B$ of two $n \times n$ - matrices $A, B$ is defined as the entry-wise product $(A * B)_{i j}:=A_{i j} B_{i j}$. Remarkably, the Hadamard product between a density matrix $\rho$ with any positive matrix $M$ of the same size $n$ defines a completely positive map:

If $M:=\sum_{j} r_{j}\left|d_{j}\right\rangle\left\langle d_{j}\right|$ is a spectral decomposition of $M$ then we may define diagonal matrices $D_{j}$ which have the coefficients of the vector $d_{j} \in \mathbb{C}^{n}$ as diagonal entries. Then the map

$$
\rho \mapsto D_{j} \rho D_{j}^{\dagger}=\left(\left|d_{j}\right\rangle\left\langle d_{j}\right|\right) * \rho
$$

is obviously completely positive. So is the positive linear combination of those maps. Channels of this type have already be considered in [11] to describe decoherence. It is clear that they commute with the Hamiltonian time evolution if the Hadamard product is calculated with respect to the energy basis. In the finite dimensional case, it we obtain also time-covariant channels by the following construction: Let $\Sigma:=\left\{\omega_{1}, \ldots, \omega_{n}\right\}$ be the eigenvalues of $H$ acting on $\mathbb{C}^{n}$. Let $\left|\omega_{j}\right\rangle$ be the corresponding eigenstates. For any $\sigma \in \mathbb{R}$ let $j_{1}, \ldots, j_{k}$ be all indices $j$ such that $\omega_{j}+\sigma$ is in the spectrum $\Sigma$. Define the "partial shift" by

$$
S_{\sigma}:=\sum\left|\omega_{j_{i}}+\sigma\right\rangle\left\langle\omega_{j_{i}}\right|
$$

where the sum runs over all spectral values $\omega$ with $\omega+\sigma \in \Sigma$.

The map $\rho \mapsto S_{\sigma} \rho S_{\sigma}^{\dagger}$ is time-covariant and also every map of the form

$$
\rho \mapsto \sum_{\sigma} S_{\sigma}\left(M_{\sigma} * \rho\right) S_{\sigma}^{\dagger}
$$

where the sum runs over all $\sigma$ for which a non-zero partial shift exists. Each $M_{\sigma}$ is an arbitrary positive matrix. It is easy to check that this map is trace-preserving if and only if

$$
\sum_{\sigma} M_{\sigma}(\omega, \omega)=1 \forall \omega \in \Sigma
$$

where the sum runs over all values $\sigma$ for which there exist an $\omega \in \Sigma$ such that $\omega+\sigma \in \Sigma$. 
In the following we will show that this is the most general form of timecovariant CP-maps. As will be shown, this holds in principle even for the infinite dimensional case with continuous spectrum when the sum is replaced by an integral over a potentially uncountable number of energy shifts.

\section{The general form of time-covariant chan- nels}

To understand our construction it is helpful to consider the finite dimensional case first. Using the Kraus representation [2]

$$
G(\rho)=\sum_{j} A_{j} \rho A_{j}^{\dagger}
$$

one may choose $A_{j}$ such that they are eigenvectors of $\alpha_{t}$ (Since the derivation for the finite dimensional case follows actually from the general derivation later in this section we only mention briefly that this can be derived as follows. One shows that the representations $t \mapsto\left[d_{k j}(t)\right]$ of $(\mathbb{R},+)$ in eq. (3) corresponding to time-translations can be choosen such that they are irreducible, i.e., one-dimensional). The eigenvalue is $\exp \left(-i \sigma_{j} t\right)$ where $\sigma_{j}$ may be any possible frequency difference $\omega-\omega^{\prime}$. For each possible value $\sigma$ we obtain a CP-map

$$
G_{\sigma}(\rho):=\sum_{\sigma=\sigma_{j}} A_{j} \rho A_{j}^{\dagger} .
$$

Let $A_{j}=U_{j}\left|A_{j}\right|$ be the polar decomposition of $A_{j}$ where the partial isometry $U_{j}$ is computed from the pseudo-inverse $\left|A_{j}\right|^{-1}$ by

$$
U_{j}:=A_{j}\left|A_{j}\right|^{-1} \text {. }
$$

One has $\alpha_{t}\left(U_{j}\right)=\exp \left(-i \sigma_{j} t\right) U_{j}$ and equivalently

$$
\left[H, U_{j}\right]=\sigma_{j} U_{j} \text { and }\left[H,\left|A_{j}\right|\right]=0
$$

since $\left|A_{j}\right|$ is $\alpha_{t}$-invariant according to $\alpha_{t}\left(A_{j}\right) \alpha_{t}\left(A_{j}^{\dagger}\right)=e^{-i \sigma_{j} t} e^{i \sigma_{j} t}$. Using equation (6) one checks easily that

$$
U_{j}|\omega\rangle=V_{j}|\omega+\sigma\rangle \forall \omega \in \Sigma
$$


where $V_{j}$ is an appropriate diagonal operator. Hence we can write

$$
A_{j}=S_{\sigma_{j}} D_{j}
$$

with the diagonal matrix $D_{j}:=\left|A_{j}\right| V_{j}$. Using the remarks of Section 5 we may write

$$
G_{\sigma}(\rho)=S_{\sigma}\left(M_{\sigma} * \rho\right) S_{\sigma}^{\dagger}
$$

where $M_{\sigma}=\sum_{j}\left|d_{j}\right\rangle\left\langle d_{j}\right|$ is defined as in Section 5 from the vectors $\left|d_{j}\right\rangle$ of diagonal entries of $D_{j}$.

We conclude:

\section{Theorem 1 (Decomposition in finite dimensions)}

Let $G$ be a CP-map on $\mathbb{C}^{n}$ which commutes with the time evolution $\alpha_{t}$ corresponding to a non-degenerate diagonal Hamiltonian. Then $G$ has the form:

$$
G(\rho)=\sum_{\sigma} S_{\sigma}\left(M_{\sigma} * \rho\right) S_{\sigma}^{\dagger}
$$

where the sum runs over all possible energy differences $\sigma$. Here $S_{\sigma}$ denotes partial shifts and $M_{\sigma}$ positive matrices.

The map $\rho \mapsto\left(M_{\sigma} * \rho\right)$ preserves the energy of every state and destroys to some extent the coherent superpositions between them. This is shown by the following two extreme cases: $M_{\sigma}=\mathbf{1}$ where $\mathbf{1}$ denotes the identity matrix. Then we have complete dephasing and obtain a mixture of energy eigenstates. If $M_{\sigma}$ has only 1 as entries it is the trivial channel which does not affect the state at all. The map $G$ consists of decohering channels followed by different energy shifts.

To see the relation of our decomposition to the transfer function note that all rank-one operators $|\omega+\sigma\rangle\langle\omega|$ span the eigenspace of $[H,$.$] with eigenvalue$ $\sigma$. A time-covariant operation has to leave this subspace invariant. Therefore one could decompose $G$ into a direct sum of linear maps $B_{\sigma}$ acting on these eigenspaces. However, in this decomposition one would have restrictions on the family $B_{\sigma}$ in order to yield a completely positive map. Therefore we have preferred a decomposition where each component is completely positive. However, the Hadamard multiplication for each frequency pair $\left(\omega, \omega^{\prime}\right)$ reminds to the multiplication with the value of the transfer function for each frequency $\omega$.

To generalize the decomposition to infinite dimensions we will need several vector-valued measures. Therefore we recall the precise definition [12]: 


\section{Definition 1 (Vector-Valued Measures and Densities)}

1. Let $\Omega$ be a set and $M$ the $\sigma$-algebra of measurable subsets of $\Omega$. For a Banach space $B$ a mapping $\gamma: M \rightarrow B$ is called a vector measure if

$$
\gamma\left(\cup_{j} m_{j}\right)=\sum_{j} \gamma\left(m_{j}\right)
$$

for all finite collections of mutually disjoint sets $m_{j}$. It is called countably additive when the same holds for countable sums.

2. Let $\nu$ be a measure on the measure space $(\Omega, M)$. A measurable function $f: \Omega \rightarrow B$ is the Radon-Nikodym derivative of $\gamma$ with respect to $\nu$ if

$$
\int_{m} f d \nu=\gamma(m) \quad \forall m \in M
$$

We also say that $f$ is the (vector-valued) density of $\gamma$ with respect to $\nu$.

For trace-class operator-valued measures we will have countable additivity in the weak sense, i.e. that the scalar measure that is given by evaluation on observables is countably additive. For CP-map valued measures we demand countable additivity only after applying the maps to states and evaluating them on observables.

In order to construct the infinite dimensional analogue of $G_{\sigma}$ we define a function

$$
f_{K, \rho}(t):=\operatorname{tr}\left(K G\left(\rho e^{-i H t}\right) e^{i H t}\right) .
$$

for every observable $K$ and state $\rho$. First consider the simple case that $G=A \rho A^{\dagger}$ where $A$ is an operator satisfying $\alpha_{t}(A)=e^{-i \sigma t} A$. Then we have

$$
f_{K, \rho}(t)=e^{i \sigma t} f_{K, \rho}(0) .
$$

If $G$ is defined by several Kraus operators $A_{j}$ which are eigenvectors with different eigenvalues $\sigma_{j}$ the function $f_{K, \rho}$ would consist of harmonic functions with all these frequencies $\sigma_{j}$. Even though we do not expect in the infinite dimensional case that we have Kraus operators which are eigenvectors of $\alpha_{t}$ it will turn out that we can nevertheless construct a decomposition of $G$ based on the Fourier transform of $f_{K, \rho}$. We show that for each positive bounded 
operator $K$ and density operator $\rho$ the function $f_{K, \rho}$ is positive semidefinite, i.e., it satisfies

$$
\sum_{k, l} x_{k} \bar{x}_{l} f\left(t_{k}-t_{l}\right) \geq 0
$$

for all vectors $x \in \mathbb{C}^{m}$ and $m$-tuples $t_{1}, \ldots, t_{m} \in \mathbb{R}$ with arbitrary $m$ :

$$
\begin{aligned}
f_{K, \rho}\left(t_{k}-t_{l}\right) & =\operatorname{tr}\left(K \sum_{j} A_{j} \rho e^{-i H\left(t_{k}-t_{l}\right)} A_{j}^{\dagger} e^{-i H\left(t_{l}-t_{k}\right)}\right) \\
& =\sum_{j} \operatorname{tr}\left(K e^{i H t_{k}} A_{j} e^{-i H t_{k}} \rho e^{i H t_{l}} A_{j}^{\dagger} e^{-i H t_{l}}\right),
\end{aligned}
$$

where we have used

$$
\sum_{j} A_{j} \rho e^{-i H t_{k}} A_{j}^{\dagger} e^{i H t_{k}}=\sum_{j} e^{i H t_{k}} A_{j} e^{-i H t_{k}} \rho A_{j}^{\dagger},
$$

which is just another version of the time-invariance condition. We have therefore

$$
\begin{aligned}
\sum_{k, l} x_{k} \bar{x}_{l} f\left(t_{k}-t_{l}\right) & =\sum_{j} \operatorname{tr}\left(K\left(\sum_{k} x_{k} e^{i H t_{k}} A_{j} e^{-i H t_{k}}\right) \rho\left(\sum_{l} \bar{x}_{l} e^{i H t_{l}} A_{j}^{\dagger} e^{-i H t_{l}}\right)\right) \\
& =\sum_{j} \operatorname{tr}\left(K C_{j} \rho C_{j}^{\dagger}\right) \geq 0
\end{aligned}
$$

with the abbreviation

$$
C_{j}:=\sum_{k} x_{k} e^{i H t_{k}} A_{j} e^{-i H t_{k}}
$$

Since $f_{K, \rho}$ is positive semidefinite it defines a unique positive scalar measure $\nu_{K, \rho}$ on $\mathbb{R}$ by Bochner's theorem [13]. For every measurable set $m \subset \mathbb{R}$ the map $K \mapsto \nu_{K, \rho}(m)$ is a positive linear functional. It is norm-continuous (with respect to the operator norm) since the norm of a positive functional is given by its value on the identity [14], i.e., by $\nu_{\mathbf{1}, \rho}(m)$. Now we restrict the functional to the set of compact operators where every norm-continuous functional is given by a trace-class operator [15]. Therefore we may define a positive trace-class operator $\rho_{m}$ by

$$
\operatorname{tr}\left(\rho_{m} K\right)=\nu_{K, \rho}(m) .
$$


Hence the map $G_{m}$ defined by $G_{m}(\rho):=\rho_{m}$ is a positive map on the traceclass operators. One can easily check that $G_{m}$ is also completely positive: one substitutes $G$ and $e^{-i H t}$ by an arbitrary tensor product extension $G \otimes i d$ by and $e^{-i H t} \otimes \mathbf{1}$ and considers $\rho$ and $K$ as operators on the extended space. Then it is obvious that the positivity argument above works similarly. We have $G_{\mathbb{R}}=G$ due to

$$
\operatorname{tr}\left(K \rho_{\mathbb{R}}\right)=\nu_{K, \rho}(\mathbb{R})=f_{K, \rho}(0)=\operatorname{tr}(K \rho) .
$$

Since the trace of each $G(\rho)$ is at most $\operatorname{tr}(G(\rho))=\operatorname{tr}(\rho)=1$, each map $G_{m}$ is a bounded operator on $T$, the set of trace-class operators. Since $G_{\mathbb{R}}$ is tracepreserving the map $m \mapsto G_{m}$ is formally an instrument in the sense of Davies (see [16], chapter 4). Think of $G_{m}(\rho) / \operatorname{tr}\left(G_{m}(\rho)\right)$ as the post-measurement state given the knowledge that the measurement outcome $\sigma$ is in $m$. Then $\rho \mapsto G(\rho)$ is formally the effect of the measurement if the measured outcome is completely ignored. Even though this interpretation refers to a virtual measurement the virtual result $\sigma$ has an observable interpretation: Assume one observes the energy of the environment before and after it has interacted with the system. Due to energy conservation $\sigma$ is the energy loss of the environment. Note that the initial energy of the environment can be observed without disturbing its state since we have assumed that it is in a stationary state. ${ }^{3}$ We shall call the probability measure $\nu_{\rho, \mathbf{1}}$ the "energy shift probabilities" in the state $\rho$.

Each map $G_{m}$ is also time-covariant: The obvious equation

$$
f_{K, \rho}=f_{\alpha_{t}(K), \alpha_{t}(\rho)}
$$

implies that it is irrelevant for the measure $\nu_{K, \rho}$ if $\alpha_{t}$ is applied to $\rho$ and $\alpha_{-t}$ to the output state (which is equivalent to applying $\alpha_{t}$ to $K$ ).

We summarize the results:

Theorem 2 For each time-covariant channel $G$ there is a unique CP-mapvalued-measure

$$
m \mapsto G_{m}
$$

such that

\footnotetext{
${ }^{3}$ The idea to observe the environment in order to have a less mixed output state (which increases information capacities) can already be found in [17, 18].
} 
1. Each $G_{m}$ is a time-covariant bounded operator on $T$.

2. The Fourier transform of $m \mapsto G_{m}$ is the function $t \mapsto \hat{G}_{t}$ with

$$
\hat{G}_{t}(\rho):=G\left(\rho e^{-i H t}\right) e^{i H t}
$$

in the sense that

$$
t \mapsto \operatorname{tr}\left(K \hat{G}_{t}(\rho)\right)
$$

is for all $K>0$ and all states $\rho$ the Fourier transform of the (nonnegative) measure

$$
m \mapsto \operatorname{tr}\left(K G_{m}(\rho)\right)
$$

The positive-map-valued measure defines a positive operator-valued-measure (POVM) [16] $m \mapsto Q_{m}$ by

$$
\operatorname{tr}\left(Q_{m} \rho\right):=\operatorname{tr}\left(G_{m}(\rho)\right) .
$$

In contrast to CP-map valued measures, POVMs describe only the probabilities for the measurement outcome without referring to the post-measurement state. The Fourier transform of this POVM will play a crucial role later.

In order to have a stronger analogy to Theorem 1 we would like to write $G$ as an integral

$$
G=\int G_{\sigma} d \nu(\sigma)
$$

with an appropriate measure $\nu$. Then the function $\sigma \mapsto G_{\sigma}$ would be the CP-map valued Radon-Nikodym derivative of the measure $m \mapsto G_{m}$, i.e., $G_{\sigma}$ would be the density of the measure with respect to the measure $\nu$. The general problem of Radon-Nikodym derivatives of instruments (CP-map valued measures) has already been considered in [19]. It is shown that it exists in the following sense:

There is a $\sigma$-finite measure $\nu$, a dense domain $\mathcal{D} \in \mathcal{H}$, and a countable family of functions

$$
\sigma \mapsto A_{k}^{\sigma}
$$

defined for $\nu$-almost all $\sigma$ such that $A_{k}^{\sigma}$ are linear operators $\mathcal{D} \rightarrow \mathcal{H}$ (not necessarily closable) such that

$$
\int \sum_{k} \| A_{k}^{\sigma}|\psi\rangle\left\|^{2}=\right\||\psi\rangle \|^{2} \quad \forall|\psi\rangle \in \mathcal{D}
$$


and

$$
\operatorname{tr}\left(K G_{m}(\rho)\right)=\int_{m} \sum_{k}\left\langle A_{k}^{\sigma} \psi \mid K A_{k}^{\sigma} \psi\right\rangle d \nu(\sigma) \quad \forall|\psi\rangle \in \mathcal{D} .
$$

Note that $A_{k}^{\sigma}$ could formally be considered as the Kraus operators of a CPmap $G_{\sigma}$ with the "only" difference that Kraus operators are not only closable but even bounded.

Equation (7) states implicitly that

$$
\sum_{k}\left\langle A_{k}^{\sigma} \psi \mid K A_{k}^{\sigma} \psi\right\rangle<\infty
$$

for $\nu$-almost all $\sigma$. Since it even converges for $K=1$ expression (8) defines a bounded positive functional on the operators $K$. We can find a unique positive trace-class operator $\rho_{\sigma}$ such that

$$
\operatorname{tr}\left(K \rho_{\sigma}\right)=\int_{m} \sum_{k}\left\langle A_{k}^{\sigma} \psi \mid K A_{k}^{\sigma} \psi\right\rangle
$$

for all compact $K$. The proof in [19] states furthermore that $\mathcal{D}$ can be the finite span of any orthogonal system of $\mathcal{H}$. Summarizing these results, we have:

\section{Theorem 3 (Radon-Nikodym derivative of the instrument)}

For every orthonormal system $\left(\left|x_{j}\right\rangle\right)$ of $\mathcal{H}$ there is a family of CP-maps $G_{\sigma}$ defined on the finite span of all rank-one operators $\left|x_{i}\right\rangle\left\langle x_{j}\right|$ such that

$$
G_{m}(\rho)=\int_{m} G_{\sigma}(\rho) d \nu(\sigma)
$$

for all $\rho$ in the domain of $G_{\sigma}$ and an appropriate $\sigma$-finite measure $\nu$.

However, the domain of the maps $G_{\sigma}$ can be extended:

\section{Lemma 1 (Extended Domains)}

Let $\rho \in T$ be arbitrary and $\nu$ ne an arbitrary measure. If the trace-class operators $G_{\sigma}(\rho)$ are consistently defined (in the sense that they define the density of the measure $\left.m \mapsto G_{m}(\rho)\right)$ then we have: 
1. The domain of $G_{\sigma}$ can consistently be extended to $\rho e^{-i H t}$ by

$$
G_{\sigma}\left(\rho e^{-i H t}\right):=G_{\sigma}(\rho) e^{-i H t} e^{i \sigma t} .
$$

Consistency means that it is the density of the measure $m \mapsto G_{m}\left(\rho e^{-i H t}\right)$. Similarly, we may define $G_{\sigma}(\exp (i H t) \rho):=\exp (-i \sigma t) \exp (i H t) G_{\sigma}(\rho)$.

2. Let $l$ be a measurable subset of $\mathbb{R}$ and $P_{l}$ be the projection onto the space of functions vanishing on the complement of $l$. Then one may extent the domain of $G_{\sigma}$ consistently to $\rho P_{l}$ by setting

$$
G_{\sigma}\left(\rho P_{l}\right):=G_{\sigma}(\rho) P_{l+\sigma}
$$

and similarly $G_{\sigma}\left(P_{l} \rho\right):=P_{l+\sigma} G_{\sigma}(\rho)$.

Proof: The Fourier transform of the measure

$$
m \mapsto \operatorname{tr}\left(K G_{m}\left(\rho e^{-i H t}\right)\right)
$$

is given by

$$
\begin{aligned}
f(r) & :=\operatorname{tr}\left(K \hat{G}_{r}\left(\rho e^{-i H t}\right)\right)=\operatorname{tr}\left(K \hat{G}_{t+r}(\rho) e^{-i H t}\right) \\
& =\int \operatorname{tr}\left(K G_{\sigma}(\rho) e^{-i H t}\right) e^{i(r+t) \sigma} d \nu(\sigma)
\end{aligned}
$$

The last equality holds since $\hat{G}_{r+t}$ is the Fourier transform of $m \mapsto G_{m}$ evaluated at $r+t$. Set $\rho_{\sigma}:=G_{\sigma}(\rho)$. We write (10) as

$$
\int \operatorname{tr}\left(K \rho_{\sigma} e^{-i H t} e^{i t \sigma}\right) e^{i r \sigma} d \nu(\sigma),
$$

which is the Fourier transform of a measure with density

$$
\sigma \mapsto \operatorname{tr}\left(K \rho_{\sigma} e^{-i H t} e^{i t \sigma}\right)
$$

This proves statement (1).

To prove (2) we have to show that

$$
\operatorname{tr}\left(K G_{m}\left(\rho P_{l}\right)\right)=\int_{m} G_{\sigma}(\rho) P_{l+\sigma} d \nu(\sigma)
$$


holds for all $K$ and measurable $m$. Consider the scalar complex-valued measure

$$
l \mapsto \operatorname{tr}\left(K G_{m}\left(\rho P_{l}\right)\right)
$$

Its Fourier transform is

$$
f(t):=\operatorname{tr}\left(K G_{m}\left(\rho e^{i H t}\right)\right)=\int_{m} \operatorname{tr}\left(K \rho_{\sigma} e^{-i H t} e^{i t \sigma}\right) d \nu(\sigma),
$$

where the last equality is due to statement (1). By

$$
e^{-i H t} e^{i t \sigma}=e^{i(H+\sigma \mathbf{1}) t}
$$

the last expression is the Fourier transform of the measure

$$
l \mapsto \int_{m} G_{\sigma}(\rho) P_{l+\sigma} d \nu(\sigma) .
$$

This proves that both sides of eq. (11) coincide.

We would like to characterize the maps $G_{\sigma}$ more explicitly. We already have done this in Theorem 1 for the finite dimensional case when the spectrum of $H$ is non-degenerate. Now we assume that $\mathcal{H}$ is the set of square integrable functions on $\mathbb{R}$

$$
\mathcal{H}:=L^{2}(\mathbb{R}, \mu),
$$

where $\mu$ is an arbitrary measure on $\mathbb{R}$ defined on the Lebesgue measurable sets. To formalize the assumption of non-degenerate spectrum we define $H$ as the multiplication operator

$$
(H \psi)(\omega)=\omega \psi(\omega) \forall \omega \in \mathbb{R} .
$$

Note that the Hadamard product can be generalized to infinite dimensions since every density operator $\rho$ has a representation $\rho=\sum_{j} p_{j}\left|\phi_{j}\right\rangle\left\langle\phi_{j}\right|$ with eigenvectors $\left|\phi_{j}\right\rangle$. Then we may interpret

$$
\rho\left(\omega, \omega^{\prime}\right):=\sum_{j} p_{j} \phi_{j}(\omega) \bar{\phi}_{j}\left(\omega^{\prime}\right)
$$

as the entries of $\rho$. This is also the representation of $\rho$ by its integral kernel, i.e.,

$$
(\rho \psi)(\omega)=\int \rho\left(\omega, \omega^{\prime}\right) \psi\left(\omega^{\prime}\right) d \mu\left(\omega^{\prime}\right) .
$$

Accordingly, we define: 


\section{Definition 2 (Hadamard product in infinite dimensions)}

For two trace-class operators $\rho, \rho^{\prime}$ and a function $M: \mathbb{R} \times \mathbb{R} \rightarrow \mathbf{C}$ we write

$$
\rho^{\prime}=M * \rho
$$

if

$$
\rho^{\prime}\left(\omega, \omega^{\prime}\right)=M\left(\omega, \omega^{\prime}\right) \rho\left(\omega, \omega^{\prime}\right) .
$$

To generalize the concept of partial shifts to $L^{2}(\mathbb{R}, \mu)$ is less straightforward. Since they should be partial isometries, the problem is two-fold: Assume first that $\mu$ is given by a non-constant and non-zero density with respect to the Lebesgue measure. Then it is intuitively clear that the generalized "shift" rescales the function to compensate the different densities at different points in order to be isometric. Assume secondly that $H$ has discrete and continuous spectrum, i.e., $\mu$ consists of singular and absolutely continuous parts with respect to the Lebesgue measure. Then one cannot expect that the discrete part of the wave function can be shifted to the continuous part and vice versa. The part of the wave function where this is the case has to be mapped to zero.

Despite of these difficulties, the following lemma shows that our concept makes sense even in the general situation:

Lemma 2 (Partial shifts) For every $\sigma \in \mathbb{R}$ there is a unique partial isometry $S_{\sigma}$ with the following properties:

1. For all $f \in \mathcal{H}$ we have

$$
\left(S_{\sigma} f\right)(\omega):=s_{\sigma}(\omega-\sigma) f(\omega-\sigma),
$$

where $s_{\sigma}$ is an appropriate measurable "scaling" function.

2. Under all partial isometries $\tilde{S}_{\sigma}$ which have a representation as in (1) with an appropriate scaling function $\tilde{s}_{\sigma}$ the operator $S_{\sigma}$ has minimal kernel, i.e., its kernel is contained in the kernel of all those $\tilde{S}_{\sigma}$.

Proof: Define the translated measure $\mu_{\sigma}(m):=\mu(m+\sigma)$. Then there is a unique decomposition

$$
\mu=\mu_{c}+\mu_{s}
$$


where $\mu_{c}$ is absolutely continuous with respect to $\mu_{\sigma}$ and $\mu_{s}$ is orthogonal to $\mu_{\sigma}[20]$. Here, orthogonality means that there is a measurable set $B$ such that $\mu_{s}(B)=0$ and $\mu_{\sigma}(\bar{B})=0$ where $\bar{B}$ denotes the complement of $B$. Let $p$ be the density of $\mu_{c}$ with respect to $\mu_{\sigma}$. Set $s_{\sigma}:=\sqrt{p}$. First we show that $S_{\sigma}$ is an isometry of the subspace $\mathcal{H}_{c}$ given by all functions $f$ with $f(\omega)=0$ for $\omega \in \bar{B}$ :

$$
\begin{aligned}
\int\left|s_{\sigma}(\omega-\sigma) f(\omega-\sigma)\right|^{2} d \mu(\omega) & =\int\left|s_{\sigma}(\omega)\right|^{2}|f(\omega)|^{2} d \mu_{\sigma}(\omega) \\
=\int|f(\omega)|^{2} p(\omega) d \mu_{\sigma}(\omega) & =\int|f(\omega)|^{2} d \mu_{c}
\end{aligned}
$$

This integral is equal to $\int|f(\omega)|^{2} d \mu$ if $f$ vanishes on $\bar{B}$. On the other hand, it is zero if $f$ vanishes on $B$. This shows that the space can be decomposed into the kernel of $S_{\sigma}$ and a subspace where $S_{\sigma}$ is an isometry. Hence we have $\left\|S_{\sigma} f\right\| \leq\|f\|$ on the whole space. Note that the equations showing this inequality show furthermore that $S_{\sigma}$ is well-defined with respect to the equivalence classes in $L^{2}(\mathbb{R}, \mu)$.

Let $\tilde{S}_{\sigma}$ be another isometry satisfying condition (1) with the scaling function $\tilde{s}_{\sigma}$. Set $D:=\tilde{s}^{-1}(0)$. Clearly, condition (1) implies $\left[H, \tilde{S}_{\sigma}\right]=\sigma \tilde{S}_{\sigma}$ and $\left[H, \tilde{S}_{\sigma}^{\dagger}\right]=-\sigma \tilde{S}_{\sigma}^{\dagger}$. Hence the initial projection $\tilde{S}_{\sigma}^{\dagger} \tilde{S}_{\sigma}$ commutes with $H$ and is therefore the multiplication operator with the characteristic function $\chi_{D}$ of a measurable set $D$. Let $f$ vanish on $\bar{D}$. Since $\tilde{S}_{\sigma}$ preserves its norm we have

$$
\int|f(\omega)|^{2} d \mu(\omega)=\int|f(\omega)|^{2} \tilde{s}_{\sigma}^{2}(\omega) d \mu_{\sigma}(\omega) .
$$

Since this holds for all functions which vanish on $\bar{D} \mu$-almost everywhere it shows that the restriction of $\mu$ to $D$ is absolutely continuous with respect to $\mu_{\sigma}$ (with the density $\tilde{s}_{\sigma}^{2}$ ). Hence the singular part of $\mu$ (with respect to $\mu_{\sigma}$ ) vanishes on $D$ and we have

$$
\int|f(\omega)|^{2} d \mu(\omega)=\int|f(\omega)|^{2} d \mu_{c}(\omega) .
$$

As can be seen in eq. (12), this shows that the norm of $f$ is also preserved by $S_{\sigma}$. Hence the isometric subspace of $\tilde{S}_{\sigma}$ is a subspace of the isometric subspace of $S_{\sigma}$ and the kernel of $S_{\sigma}$ is a subspace of the kernel of $\tilde{S}_{\sigma}$.

Fortunately, these shifts have the following property: 
Lemma 3 The partial shift of Lemma 2 satisfy

$$
S_{\sigma}^{\dagger}=S_{-\sigma} .
$$

Proof: Rewriting the inner product of $\mathcal{H}$ as an integral one checks easily that $S_{\sigma}^{\dagger}$ has to be a translation by $-\sigma$ with an appropriate scaling function. It is easy to see that $S_{-\sigma} S_{\sigma}=S_{\sigma}^{\dagger} S_{\sigma}$ : On the kernel of $S_{\sigma}$ both products coincide trivially. On its orthogonal complement, i.e., the isometric subscape of $S_{\sigma}$ the operator $S_{\sigma}^{\dagger} S_{\sigma}$ is the identity. But this must also be true for $S_{-\sigma} S_{\sigma}$ because the isometric subspace of $S_{-\sigma}$ must contain the isometric subspace of $S_{\sigma}^{\dagger}$ since $S_{-\sigma}$ has minimal kernel in the set of all partially isometric translations (see property (2) in Lemma 2). Using general properties of partial isometries the image of $S_{\sigma}^{\dagger}$ satisfies therefore

$$
\operatorname{Im} S_{\sigma}^{\dagger}=\operatorname{Im}\left(S_{\sigma}^{\dagger} S_{\sigma}\right)=\operatorname{Im}\left(S_{-\sigma} S_{\sigma}\right) \leq \operatorname{Im} S_{-\sigma} .
$$

Reversing this inequality by taking the orthogonal complement we obtain

$$
\operatorname{ker} S_{\sigma} \geq \operatorname{ker} S_{-\sigma}^{\dagger} .
$$

Because this holds for all $\sigma \in \mathbb{R}$ we have

$$
k e r S_{-\sigma} \geq \operatorname{ker} S_{\sigma}^{\dagger} .
$$

Since $S_{-\sigma}$ has minimal kernel we conclude

$$
k e r S_{-\sigma}=k e r S_{\sigma}^{\dagger} .
$$

Due to the uniqueness of the maximal element in the set of all partially isometric translations we have

$$
S_{\sigma}^{\dagger}=S_{-\sigma} .
$$

Note that we can only write $G_{\sigma}(\rho)$ in the form $S_{\sigma}\left(M_{\sigma} * \rho\right) S_{\sigma}^{\dagger}$ if the density operator $G_{\sigma}(\rho)$ acts only on the image of $S_{\sigma}$ (which coincides with the image of the projection $S_{\sigma} S_{\sigma}^{\dagger}$ ). But this is indeed the case:

Lemma 4 If $\rho$ is in the domain of $G_{\sigma}$ its output $\rho_{\sigma}:=G_{\sigma}(\rho)$ satisfies

$$
S_{\sigma} S_{\sigma}^{\dagger} \rho_{\sigma} S_{\sigma} S_{\sigma}^{\dagger}=\rho_{\sigma} .
$$


Proof: We will show $G_{\sigma}(\rho) S_{\sigma} S_{\sigma}^{\dagger}=G_{\sigma}(\rho)$. The statement $S_{\sigma} S_{\sigma}^{\dagger} G_{\sigma}(\rho)=$ $G_{\sigma}(\rho)$ follows similarly. Due to Lemma 3 we have $S_{\sigma} S_{\sigma}^{\dagger}=S_{-\sigma}^{\dagger} S_{-\sigma}$, which is the projection onto the isometric subspace of $S_{-\sigma}$. It can explictly be given as follows (see proof of Lemma 2): Let $\mu:=\mu_{c} \oplus \mu_{s}$ be the decomposition into the absolutely continuous and singular part of $\mu$ with respect to $\mu_{-\sigma}$. Let $C$ be a set such that $\mu_{-\sigma}(\bar{C})=0$ and $\mu_{s}(C)=0$. Then $S_{-\sigma}^{\dagger} S_{-\sigma}=P_{C}$. Due to $\mu(\bar{C}-\sigma)=\mu_{-\sigma}(\bar{C})=0$ we have $P_{\bar{C}-\sigma}=0$. This implies

$$
G_{\sigma}(\rho) P_{\bar{C}}=G_{\sigma}\left(\rho P_{\bar{C}-\sigma}\right)=0 .
$$

For the construction in the sequel we choose the ONS in Theorem 3 such that its finite span contains a vector $|\psi\rangle$ with $\psi(\omega) \neq 0$ for $\mu$-almost all $\omega$.

Now we can define a function

$$
M_{\sigma}: \mathbb{R} \times \mathbb{R} \rightarrow \mathbf{C}
$$

by

$$
M_{\sigma}\left(\omega, \omega^{\prime}\right):=\frac{k\left(\omega, \omega^{\prime}\right)}{\psi(\omega) \bar{\psi}\left(\omega^{\prime}\right)}
$$

where the function $k$ represents the trace-class operator $S_{\sigma}^{\dagger} \rho_{\sigma} S_{\sigma}$. Even though this function is not well-defined, the calculations below show that the freedom of choosing $M_{\sigma}$ is irrelevant.

By construction and using Lemma 4, we have

$$
G_{\sigma}(\rho)=\rho_{\sigma}=S_{\sigma}\left(M_{\sigma} * \rho_{\sigma}\right) S_{\sigma}^{\dagger} .
$$

We conclude

$$
G(|\psi\rangle\langle\psi|)=\int S_{\sigma}\left(M_{\sigma} *(|\psi\rangle\langle\psi|)\right) S_{\sigma}^{\dagger} d \nu(\sigma)
$$

with $|\psi\rangle$ as above. Using Lemma 1, part (1), we have

$$
\int G_{\sigma}\left(e^{-i H t}|\psi\rangle\langle\psi| e^{i H s}\right) d \nu(\sigma)=\int S_{\sigma}\left(M_{\sigma} *\left(e^{-i H t}|\psi\rangle\langle\psi| e^{i H s}\right)\right) S_{\sigma}^{\dagger} d \nu(\sigma),
$$

for all $s, t \in \mathbb{R}$. The finite span of these rank-one operators is dense in $T$ since $H$ is the multiplication with the identity and the span of all $\exp (-i H t)|\psi\rangle$ is therefore dense in $\mathcal{H}$. We conclude: 


\section{Theorem 4 (Dephasing - Energy Shift Representation)}

There is a family of functions $\left(M_{\sigma}\right)_{\sigma \in \mathbb{R}}$

$$
M_{\sigma}: \mathbb{R} \times \mathbb{R} \rightarrow \mathbf{C},
$$

and a $\sigma$-finite measure $\nu$ on $\mathbb{R}$ such that for a dense set of density operators $\rho$ the decompostion

$$
G(\rho)=\int S_{\sigma}\left(M_{\sigma} * \rho\right) S_{\sigma}^{\dagger} d \nu(\sigma)
$$

holds.

We would like to have an analogy to the statement that $M_{\sigma}$ is in the finite dimensional case a positive matrix. However, we have specified $M_{\sigma}$ only as a function and not as an operator. It seems straightforward to consider it as an operator by

$$
\left(M_{\sigma} \psi\right)(\omega):=\int M_{\sigma}\left(\omega, \omega^{\prime}\right) \psi\left(\omega^{\prime}\right) d \mu\left(\omega^{\prime}\right) .
$$

But it is easy to see that this is not in general well-defined. Let, for instance, $G$ be the identity on the density operators on $l^{2}(\mathbb{Z})$. Then the decomposition of $G$ reduces to $G(\rho)=M_{0} * \rho$ with $M_{0}\left(\omega, \omega^{\prime}\right)=1$. The formal matrix multiplication of this "all-one" matrix with any square-integrable sequence leads to infinite coefficients. However, formally it is like a positive operator in the following sense.

$$
M_{\sigma} *(|\psi\rangle\langle\psi|)
$$

is by construction the trace-class operator $\rho_{\sigma}$. Translated to finite dimensions this expression to

$$
D M_{\sigma} D^{\dagger}
$$

where $D$ is a non-singular diagonal matrix (in analogy to the statement that $\psi(\omega)$ vanishes almost nowhere. Such a matrix can only be positive if $M_{\sigma}$ is itself positive. In this sense we consider $M_{\sigma}$ as positive even though it is not an operator. In analogy to the finite dimensional case, an exact measurement of the energy of the environment (before and after the interaction has taken place) would lead to a map

$$
S_{\sigma}\left(M_{\sigma} * \rho\right) S_{\sigma}^{\dagger},
$$


which is only a dephasing channel up to the known energy shift.

It should be noted that (depending on $\nu$ ) the integral may represent a finite, or countable infinite sum, or a continuous integral even though the Kraus representation of $G$ is always possible with a countable sum [2].

\section{Superpositions between states in the same orbit}

The "Hadamard-channel" $\rho \mapsto M * \rho$ with a positive matrix $M$ allows perfect classical information transfer by using energy eigenstates as logical states. Its quantum capacity depends on the destruction of off-diagonal elements, i.e., whether the output states are more or less stationary states. Explicitly one has the following lower bound on the quantum capacity:

Lemma 5 In dimension $n$, the quantum capacity $Q$ of the channel $\rho \mapsto M * \rho$ with $M(\omega, \omega)=1$ for all $\omega \in \Omega$ satisfies

$$
Q \geq \log _{2}(n)-S(M / n)
$$

where $S($.$) denotes the von-Neumann entropy.$

Proof: Let

$$
M:=\sum_{j}\left|m_{j}\right\rangle\left\langle m_{j}\right|
$$

be a decomposition into mutually orthogonal rank-one operators with nonnormalized vectors $\left|m_{j}\right\rangle$. Let $D_{j}$ be the diagonal matrices having the coefficients of $\left|m_{j}\right\rangle$ as entries. Then we have

$$
G(\rho)=\sum_{j} D_{j} \rho D_{j}^{\dagger} .
$$

The quantum capacity is given by the maximum of the coherent information [23]. Let $|\phi\rangle\langle\phi|$ a state on $\mathbb{C}^{n} \otimes \mathbb{C}^{n}$ and $\rho$ its restriction to the right component. Then the coherent information is defined as

$$
S(G(\rho))-S(i d \otimes G)(|\phi\rangle\langle\phi|),
$$


where $S$ denotes the von-Neumann entropy. Consider the maximally entangled state

$$
|\phi\rangle:=\frac{1}{\sqrt{n}} \sum_{j}|j\rangle \otimes|j\rangle .
$$

Note that $\rho$ is the maximally mixed state and that it is preserved by the channel. Hence we have $G(S(\rho))=\log _{2}(n)$ and

$$
(i d \otimes G)(|\phi\rangle\langle\phi|)=\sum_{j}\left(1 \otimes D_{j}\right)|\phi\rangle\langle\phi|\left(1 \otimes D_{j}^{\dagger}\right) .
$$

Furthermore we have

$$
\left\langle\phi\left|\left(1 \otimes D_{j}^{\dagger}\right)\left(1 \otimes D_{i}\right)\right| \phi\right\rangle=\operatorname{tr}\left(D_{j}^{\dagger} D_{i}\right)=\left\langle m_{j} \mid m_{i}\right\rangle=0 .
$$

Therefore the sum (14) is already the spectral decomposition of $(i d \otimes G)(|\phi\rangle\langle\phi|)$. The eigenvalues are the square of the length of each vector $\left(1 \otimes D_{j}\right)|\phi\rangle$. It is given by $\left\langle m_{j} \mid m_{j}\right\rangle / n$. Hence the entropy of the output state is the vonNeumann entropy $S(M / n)$.

Here the destruction of quantum information coincides with the destruction of timing information in the sense that states on the same orbit of $\alpha_{t}$ become less distinguishable. It is obvious that decoherence with respect to the energy eigenbasis leads destroys always timing information in this sense. Here we want to address the question what happens when one considers orthogonal states on the same orbit of the time evolution as reference basis. Is it possible that a time-covariant channel destroys superpositions between them without affecting the basis states? Consider two vector states $\left|\phi_{0}\right\rangle$ and $\left|\phi_{s}\right\rangle:=\exp (-i H s)\left|\phi_{0}\right\rangle$. Clearly, $G\left(\left|\phi_{0}\right\rangle\left\langle\phi_{0}\right|\right)=\left|\phi_{0}\right\rangle\left\langle\phi_{0}\right|$ implies

$$
S_{\sigma}\left(M_{\sigma} *\left(\left|\phi_{0}\right\rangle\left\langle\phi_{0}\right|\right)\right) S_{\sigma}^{\dagger}=0
$$

for all $\sigma \neq 0$. This follows from the observation that the output state can only be pure if all output operators are linearly dependent. Every $\sigma \neq 0$ would lead to a different output state. Furthermore we have

$$
M_{0} *\left(\left|\phi_{0}\right\rangle\left\langle\phi_{s}\right|\right)=M_{0} *\left(\left|\phi_{0}\right\rangle\left\langle\phi_{0}\right|\right) e^{i H s}=\left|\phi_{0}\right\rangle\left\langle\phi_{s}\right| .
$$

One can conclude easily that every density operator acting on the subspace spanned by $\left|\phi_{0}\right\rangle$ and $\left|\phi_{s}\right\rangle$ is preserved by $G$. A little bit more general, one has: 
Theorem 5 Let $G$ be a time-covariant CP-map on a system with non-degenerated Hamiltonian. If there is any pure state $|\phi\rangle\langle\phi|$ with

$$
G(|\phi\rangle\langle\phi|)=|\phi\rangle\langle\phi|
$$

then $G$ leaves all density operators invariant which act on the Hilbert subspace spanned by all $\exp (-i H t)|\phi\rangle$.

The essential argument above is that there exist no states which are invariant with respect to $S_{\sigma}$ for some $\sigma \neq 0$. An analogue statement would not be true for covariance with respect to a discretized time evolution:

Consider the Hilbert space $l^{2}(\mathbb{Z})$ of square summable two-sided sequences and the discrete time evolution given by the translation $(U \psi)(n):=\psi(n+1)$. Let $\left(\left|e_{n}\right\rangle\right)_{n \in \mathbb{Z}}$ be the canaonical basis of $l^{2}(\mathbb{Z})$. Then the channel

$$
\rho \mapsto \sum_{n \in \mathbb{Z}}\left|e_{n}\right\rangle\left\langle e_{n}|\rho| e_{n}\right\rangle\left\langle e_{n}\right|
$$

leaves all basis states $\left|e_{n}\right\rangle$ invariant but destroys all superpositions.

The assumption that a pure state is preserved by $G$ is rather strong. Actually we want to figure out whether distiguishability of different states in the same orbit may be conserved even though their superpositions are distroyed. We consider the following extreme case:

\section{Definition 3 (Reliable timing)}

A time-covariant channel G has the "reliable timing property" (with respect to the time $s$ ) if there exists an input density operator $\rho$ and a real number $s$ such that for $\rho_{s}:=\alpha_{s}(\rho)$ the outputs $G(\rho)$ and $G\left(\rho_{s}\right)$ are perfectly distinguishable, i.e., the density matrices are mutually orthogonal.

It is easy to verify that the input state $\rho$ can be chosen to be pure. To justify the definition, we show that this property appears in the following situation: Assume a sender, say Alice, wants to send a signal to a receiver, say Bob. Assume furthermore that it should be guaranteed that Bob receives the signal in the time interval $\left[t_{1}, t_{2}\right]$. This requires that the physical state $\rho$ of the signal (when it is sent) is perfectly distinguishable from the time evolved state $\rho_{s}$ with $s:=t_{1}-t_{2}$. This is due to the fact that the following "measurement" distinguishes between them: wait the time $t_{1}$ and ask Bob 
whether he has already received the signal. If the medium between Alice and Bob modifies the signal, we may model this by a time-covariant operation $G$ which has clearly to preserve the distinguishability of the states $\rho$ and $\rho_{s}$.

It is clear that reliable transfer of classical information requires two states $\rho$ and $\gamma$ such that $G(\rho)$ and $G(\gamma)$ are mutually orthogonal. The remark above shows that there are situations in classical information processing where two orthogonal output states should exists on the same orbit since reliable timing requires this feature. To see that non-trivial channels with this property exist one may construct a channel of the form

$$
\rho \mapsto \sum_{j} p_{j} S_{\sigma_{j}} \rho S_{\sigma_{j}}^{\dagger}
$$

where the $\sigma_{j}$ are chosen such that $\left\langle\phi \mid S_{\sigma_{i}}^{\dagger} S_{\sigma_{j}} \phi\right\rangle=0$ for $i \neq j$. This condition is, for instance, satisfied if the minimal distance between the values $\sigma_{j}$ exceeds the spectral bandwidth of the input state. Real physical channels will satisfy condition 3 at most approximatively.

In order to consider the quantum capacity of reliable timing channels we choose $\rho$ and $\rho_{s}=U_{s} \rho U_{s}^{\dagger}$ with $U_{s}:=\exp (-i H s)$ such that $\rho$ and $\rho_{s}$ are mutually orthogonal. For simplicity we assume that the dynamical evolution of $G(\rho)$ is periodic. We obtain a set

$$
G\left(\rho_{0}\right), G\left(\rho_{s}\right), G\left(\rho_{2 s}\right), \ldots, G\left(\rho_{s(N-1)}\right)
$$

of $N$ mutually perfectly distinguishable density matrices. Let $P$ be the support of $G(\rho)$, i.e. the smallest projection with $P G(\rho)=G(\rho)$. Then the projections $U_{s j} P U_{s j}^{\dagger}$ with $j=0, \ldots, N-1$ are mutually orthogonal.

Now we consider the channel with input

$$
\mathbb{C}^{N} \equiv \operatorname{span}\left\{\left|\phi_{s j}\right\rangle\right\}_{j=0, \ldots N-1}
$$

It is clear that there exist pure input states $\left|\psi_{0}\right\rangle, \ldots,\left|\psi_{s(N-1)}\right\rangle$ for which the orthogonality of the output states is satisfied. By sending either of these $N$ states $G$ can transfer $\log _{2} N$ bits of classical information. We want to derive sufficient conditions under which $G$ allows also to send superpositions between the chosen basis states. For doing so, we restrict $G$ to a channel $K$ on $N \times N$-density matrices as follows. 
The input is restricted to the span of all $\left|\psi_{s j}\right\rangle$ with $j=0, \ldots, N-1$. The corresponding output space is

$$
\mathcal{H}_{r}:=\oplus_{j=0}^{N-1} U_{s j} P U_{s j}^{\dagger} \mathcal{H} .
$$

We may consider this space as the tensor product

$$
\mathcal{H}_{r}=\mathbb{C}^{N} \otimes P \mathcal{H}
$$

if we identify the spaces $U_{s j} P U_{s j}^{\dagger} \mathcal{H}$ for $j \neq 0$ with $P \mathcal{H}$ via arbitrary unitaries. It is straightforward and convenient to chose the isomorphisms $U_{s j}$ with $j=$ $0, \ldots, N-1$. For any output density operator $\gamma$ acting on $\mathcal{H}_{r}$ the entry corresponding to $|j\rangle\langle k|$ of its restriction to $\mathbb{C}^{N}$ is given by

$$
\operatorname{tr}\left(U_{s k} P U_{s j}^{\dagger} \gamma\right) .
$$

To determine the channel $K$ we have to compute all values

$$
\operatorname{tr}\left(U_{s k} P U_{s j}^{\dagger} G\left(\left|\phi_{s l}\right\rangle\left\langle\phi_{s m}\right|\right)\right),
$$

for $k, j, l, m \in\{0, \ldots, N-1\}$. Due to the reliable timing property we know that each state $\left|\phi_{s j}\right\rangle\left\langle\phi_{s j}\right|$ leads to the output state $|j\rangle\langle j|$. Furthermore the operator $\left|\phi_{s l}\right\rangle\left\langle\phi_{s m}\right|$ leads with certainty to a multiple of $|l\rangle\langle m|$. Roughly speaking, the reason is that a CP-map which maps the states $|m\rangle\langle m|$ and $|l\rangle\langle l|$ onto itself maps also $|l\rangle\langle m|$ onto multiples of itself. This could, for instance, be shown by reformulating the CP-map as the restriction of an appropriate unitary map (see [14]). Hence we have only to determine which factors the off-diagonal terms obtain. Due to the symmetry of the channel with respect to time translations $U_{s j}$ we have only to evaluate

$$
v(j):=\operatorname{tr}\left(U_{s j} P G\left(\left|\phi_{0}\right\rangle\left\langle\phi_{s j}\right|\right)\right)=\operatorname{tr}\left(P G\left(\left|\phi_{0}\right\rangle\left\langle\phi_{s j}\right| U_{s j}\right)\right) .
$$

Then $K$ is explicitly given by

$$
K\left(\sum_{j k} c_{j k}|j\rangle\langle k|\right)=\sum_{j k} c_{j k} v(j-k)|j\rangle\langle k|,
$$

This shows that the channel $K$ is a "Hadamard channel" even though we have not chosen the energy states as reference basis but the states $\left|\phi_{s j}\right\rangle$ instead. Furthermore, $K$ is given by Hadamard multiplication with a circulant matrix 
$V$ with entries $V_{i j}:=v(i-j)$. In order to calculate $v$ we use the explicit form of $G$ according to Theorem 3. Using Lemma 1 this yields

$$
v(j)=\int \operatorname{tr}\left(G_{\sigma}\left(\left|\phi_{0}\right\rangle\left\langle\phi_{0}\right|\right) e^{-i \sigma s j} d \nu(\sigma)=f_{\left|\phi_{0}\right\rangle\left\langle\phi_{0}\right|, \mathbf{1}}(-s j),\right.
$$

i.e., the Fourier transform of the energy shift probability measure $\nu_{\left|\phi_{0}\right\rangle\left\langle\phi_{0}\right|, \mathbf{1}}$ evaluated at the points $-s j$. The eigenvalues $q_{0}, \ldots, q_{M-1}$ of $V / n$ are given by the inverse Fourier transform of $v$ :

$$
q_{k}:=\frac{1}{N} \sum_{j=0}^{N-1} v(j) e^{-i j k}
$$

Using Lemma 5 we conclude:

\section{Theorem 6 (Q-capacity of channels with reliable timing)}

Let $G$ be a time-covariant channel with reliable timing property with respect to the time $s$ and the initial state $\left|\phi_{0}\right\rangle$. Let the dynamics be periodic with period length $s N$. Then the quantum capacity can only be zero if the Fourier transform $\hat{p}$ of the probability measure $p(m):=\operatorname{tr}\left(G_{m}\left(\left|\phi_{0}\right\rangle\left\langle\phi_{0}\right|\right)\right)$ has zeros at sj for $j=1, \ldots, N-1$. Otherwise the quantum capacity is at least

$$
\log _{2} N-\sum_{k} q_{k} \log _{2} q_{k},
$$

where $q=\left(q_{0}, \ldots, q_{N-1}\right)$ is the discrete Fourier transform of the evaluation of $f_{\left|\phi_{0}\right\rangle\left\langle\phi_{0}\right|, 1}$ on the $N$ points $0,-s,-2 s, \ldots,-(N-1) s$.

Of course there are many possibilities to define finite dimensional channels from the original one. However, to study whether $G$ destroys superpositions between different states in the orbit ("different pointer states of a clock") one has always to consider

$$
G\left(\left|\phi_{0}\right\rangle\left\langle\phi_{s j}\right|\right)=\hat{G}_{-s j}\left(\left|\phi_{0}\right\rangle\left\langle\phi_{0}\right|\right) e^{i H s j} .
$$

This shows that the Fourier transform of the measure $\left(G_{m}\right)$ is decisive. For deriving the lower bound above we have evaluated it for the observable $\mathbf{1}$ which leads to the Fourier transform of the POVM $\left(Q_{m}\right)$ defined in the last section. However, $\mathbf{1}$ is not necessarily optimal for detecting superpositions between the output states. 


\section{Gaussian channel on a single mode}

The Hilbert space of a single mode in quantum optics is $l^{2}\left(\mathbb{N}_{0}\right)$, the set of square summable sequences. It can also be interpreted as the energy levels of a harmonic oscillator. A time-covariant channel which is often considered is the following. Using position and momentum observables $X$ and $P$, respectively, we define a translation $\left(a_{1}, a_{2}\right) \in \mathbb{R}^{2}$ by the unitary transformation $\exp \left(i\left(a_{1} X+a_{2} P\right)\right)$, where $X:=a^{\dagger}+a$ and $P:=\left(a^{\dagger}-a\right) / i$. It is convenient to introduce variables $r \in \mathbb{R}_{0}^{+}$and $z$ on the complex unit circle $\Gamma$ by $a_{1}+i a_{2}=r z$. Rewriting the translation with operators $a^{\dagger}$ and $a$ and the parameters $z, r$ we obtain the translation operator (compare [24]) by

$$
D(z, r):=\exp \left(r\left(\bar{z} a^{\dagger}-z a\right)\right)=e^{i r^{2} / 2} e^{r \bar{z} a^{\dagger}} e^{-r z a} .
$$

Since the global phase factor is irrelevant we will, in abuse of notation, use $D(z, r)$ for the term without this factor.

Let the gaussian channel be given by a random displacement $r z$ according to the two-dimensional rotation invariant gauss distribution. Note that rotation symmetry is necessary in order to obtain a time-covariant channel since the Hamiltonian $H:=\operatorname{diag}(0,1,2, \ldots)$ corresponds to a rotation in the "phase space". Note that the possible energy shifts are in $\mathbb{Z}$, hence we expect a countable sum of $G_{\sigma}$ which are (in contrary to the general case) not only densely defined.

The whole channel is given by (see [25])

$$
G(\rho)=\iint_{\Gamma} D(z, r) \rho D(\bar{z}, r) d z p(r) d r
$$

with $p(r):=\exp \left(-r^{2} /\left(2 s^{2}\right)\right) r / s^{2}$ and $s$ denotes the standard deviation.

As usual, we introduce the creation operator $a^{\dagger}$ and the annihilation operator $a$ by $a|j\rangle:=\sqrt{j}|j-1\rangle$ for $j \geq 1$ and $a|0\rangle=0$. Then we write $D(z, r)$ as the power series

$$
D(z, r)=e^{r \bar{z} a^{\dagger}} e^{-r z a}=\sum_{m \geq 0} \frac{(r \bar{z})^{n}\left(a^{\dagger}\right)^{m}}{m !} \sum_{n \geq 0} \frac{(-r z)^{n} a^{n}}{n !}
$$

It decomposes canonically into terms $D_{\sigma}(z, r)$ with $\sigma \in \mathbb{Z}$ satisfying the commutation relation $\left[H, D_{\sigma}(z, r)\right]=\sigma D_{\sigma}(z, r)$ if we define

$D_{\sigma}(z, r):=\sum_{n \geq-\sigma, 0} \frac{(r \bar{z})^{n+\sigma}\left(a^{\dagger}\right)^{n+\sigma}}{(n+\sigma) !} \frac{(-r z)^{n} a^{n}}{n !}=\bar{z}^{\sigma} r^{\sigma} \sum_{n \geq-\sigma, 0}(-1)^{n} \frac{\left(a^{\dagger}\right)^{n+\sigma}}{(n+\sigma) !} \frac{a^{n}}{n !}\left(r^{2}\right)^{n}$. 
Note that $D_{\sigma}(z, r)$ maps states with energy $j$ onto states with energy $j+\sigma$. This suggests already that they may correspond to the maps $G_{\sigma}$ of Section 6. The operator

$$
D_{\sigma}(r):=D_{\sigma}(z, r) z^{\sigma} \sqrt{2 \pi}
$$

is independent of $z$. We conclude therefore that terms of the form $D_{\sigma}(\bar{z}, r) \rho D_{\sigma^{\prime}}(z, r)$ cancel for $\sigma \neq \sigma^{\prime}$ after integration over all $z \in \Gamma$. We obtain

$$
G(\rho)=\sum_{\sigma \in \mathbb{Z}} \int D_{\sigma}(r) \rho D_{\sigma}(r) p(r) d r .
$$

Consider the case $\sigma \geq 0$ first. Then we have

$$
\begin{aligned}
\left(a^{\dagger}\right)^{n+\sigma} a^{n}|j\rangle & =\sqrt{(j+\sigma) \ldots(j+1)} j(j-1) \ldots(j-n+1)|j+\sigma\rangle \\
& =\frac{1}{\sqrt{(j+1) \ldots(j+\sigma)}} \frac{(j+\sigma) !}{(j-n) !}|j+\sigma\rangle,
\end{aligned}
$$

for all $n \leq j$. For the case $\sigma<0$ we obtain

$$
\left(a^{\dagger}\right)^{n+\sigma} a^{n}|j\rangle=\sqrt{j(j-1) \ldots(j+\sigma+1)} \frac{(j+\sigma) !}{(j-n) !}|j+\sigma\rangle,
$$

For $\sigma \geq 0$ we conclude

$$
\begin{aligned}
D_{\sigma}(r)|j\rangle & =\frac{r^{\sigma}}{\sqrt{(j+1) \ldots(j+\sigma)}} \sum_{n=0}^{j}(-1)^{n} \frac{(j+\sigma) !}{(j-n) !(n+\sigma) ! n !}\left(r^{2}\right)^{n}|j+\sigma\rangle \\
& =\frac{r^{\sigma}}{\sqrt{(j+1) \ldots(j+\sigma)}} L_{j}^{\sigma}\left(r^{2}\right)|j+\sigma\rangle,
\end{aligned}
$$

where

$$
L_{j}^{(\sigma)}(x):=\sum_{n=0}^{j}(-1)^{n} \frac{(j+\sigma) !}{(j-n) !(n+\sigma) ! n !}(x)
$$

is a Laguerre polynomial [26].

Hence we have

$$
M_{\sigma}\left(j, j^{\prime}\right)=\int \frac{\left(r^{2}\right)^{\sigma}}{\sqrt{(j+1) \ldots(j+\sigma)\left(j^{\prime}+1\right) \ldots\left(j^{\prime}+\sigma\right)}} L_{j}^{(\sigma)}\left(r^{2}\right) L_{j^{\prime}}^{(\sigma)}\left(r^{2}\right) p(r) d r .
$$

For negative $\sigma$ we may express $M_{\sigma}$ similarly by Laguerre polynomials together with a different factor. Hence the decomposition of the single mode gaussian channel can be given in a closed form even though it leads to a less familiar representation. 


\section{Conclusions}

We have shown that every time-covariant CP-map has a representation as an integral over a family of CP-maps. If the spectrum of the system Hamiltonian is non-degenerated, each of these components consists of a Hadamard multiplication with a positive operator followed by an energy shift. Formally, the output of the channel is an unselected post-measurement state. The measured (and ignored) quantity is the energy which has been transferred to or from the environment. Conditional to the measurement outcome a different dephasing channel is applied.

Furthermore we have addressed the question to what extent covariant channels can destroy superpositions between $N$ mutually orthogonal states on the same orbit of the time evolution. For a specific type of channels (with "reliable timing property") we have shown that the general decomposition helps to derive lower bounds on the quantum capacity.

The decomposition presented here may be a helpful approach to describe a rather general type of decoherence and relaxation phenomena.

We have calculated the decomposition explicitly for a rotation invariant gaussian channel acting on the state space of a single mode Fock space. The dephasing operations can then be described using Laguerre polynomials.

\section{Acknowledgments}

Thanks to Jürgen Schweizer for useful discussions and Leonid Gurvitz for a helpful remark. This work has been supported by the DFG-project "Komplexität und Energie" in the SPP VIVA. Part of this work has been done during a visit at Los Alamos National Laboratory.

\section{References}

[1] W. Wootters and W. Zurek. A single quantum bit can not be cloned. Nature, page 802, 1982.

[2] K. Kraus. States, effects, and operations: Fundamental notions in quantum theory. Springer-Verlag, Berlin, 1983. 
[3] A. Holevo. Remarks on the classical capacity of quantum channels. quant-ph/0212025.

[4] S. Barut and R. Rạczka. Theory of group representations and applications. PSP, Warszawa, 1977.

[5] A. Holevo. Covariant quantum markovian semigroups. J. Math. Phys., 37(4):1812, 1996.

[6] D. Janzing and T. Beth. Quasi-order of clocks and their synchronism and quantum bounds for copying timing information. IEEE Trans. Inform. Theor., 2003, 49(1):230-240, 2003.

[7] L. Zadeh and C. Desoer. Linear System Theory. Mc-Graw Hill, 1963.

[8] H. Baumgärtel and M. Wollenberg. Mathematical Scattering theory. Birkhäuser, Basel, 1983.

[9] D. Yafaev. Mathematical Scattering Theory. Amer. Math. Soc., Providence, 1992.

[10] B. Kümmerer and H. Maassen. A scattering theory for markov chains. Inf. Dim. Analysis, Quant. Prob. and Rel. Topics, (3), 2000.

[11] T. Havel, Y. Sharf, L. Viola, and D. Cory. Hadamard products of product operators and the design of gradient-diffusion experiments for simulating decoherence by nmr spectroscopy. quant-ph/000901, 2000.

[12] J. Diestel and J. Uhl. Vector Measures. Math. Surveys No. 15, Am. Math. Soc. 1977.

[13] A. Knapp. Group Representations and Harmonic Analysis, volume Part II. Not. Amer. Math. Soc, 1996.

[14] V. Paulsen. Completely bounded maps and dilations. Longman Scientific Technical, New York, 1986.

[15] P. Marchetti and R. Rubele. Quantum logic and non-commutative geometry. quant-ph/0405159. 
[16] E. Davies. Information and quantum measurement. IEEE Trans. Inform. Theory, IT-24(5):596-599, 1978.

[17] M. Gregoratti and R. Werner. On quantum error-correction by classical feedback in discrete time. quant-ph/0403092.

[18] P. Hayden and C. King. Correcting quantum channels by measuring the environment. quant-ph/0409026.

[19] A. Holevo. Radon-Nikodym derivatives of quantum instruments. Journ. Math. Phys., vol.39, No.3, 1373.

[20] M. Rao. Measure Theory and Integration. John Wiley \& Sons, New York, 1987.

[21] G. Murphy. $C^{*}$-algebras and operator theory. Academic Press, Boston, 1990.

[22] J. Conway. A Course in Functional Analysis. Springer, 1990.

[23] I. Devetak. The private classical capacity and quantum capacity of a quantum channel. quant-ph/0304127, 2003.

[24] L. Davidovich. Quantum optics in cavities, phase space representations, and the classical limit of quantum mechanics. AIP Conference Proceedings Vol 464, (1):3, 1999.

[25] V. Giovannetti and S. Lloyd. Additivity properties of a gaussian channel. Phys. Rev. A, 69, 2004.

[26] H. Buchholz. The confluent hypergeometric function. Springer, Berlin, 1969. 